\title{
Condensation Polymers of Terephthalic Acid and 1,4-Diaminobutane and Their Schiff Base Complexes
}

\author{
Sandeep Rai, ${ }^{1}$ Anjali Bajpai, ${ }^{2}$ and Snehal Lokhandwala ${ }^{1}$ \\ ${ }^{1}$ Shroff S. R. Rotary Institute of Chemical Technology, Block No. 402, At \& Post Vataria, \\ District Bharuch, Gujarat 393002, India \\ ${ }^{2}$ Department of Chemistry, Government Autonomous Model Science College, Jabalpur 482001, India
}

Correspondence should be addressed to Sandeep Rai; sadeep1964@yahoo.co.in

Received 12 June 2013; Accepted 1 July 2013

Academic Editor: Yves Grohens

Copyright (C) 2013 Sandeep Rai et al. This is an open access article distributed under the Creative Commons Attribution License, which permits unrestricted use, distribution, and reproduction in any medium, provided the original work is properly cited.

\begin{abstract}
Amino-terminated oligomeric poly(tetramethylene terephthalamide) (PTTA) was prepared by condensation of terephthalic acid and 1,4-diaminobutane using phosphorylation technique. Schiff base complexes of this polyamide were synthesized with salicylaldehyde and 2-hydroxy-1-naphthaldehyde complexes of $\mathrm{Co}(\mathrm{II}), \mathrm{Ni}(\mathrm{II})$, and $\mathrm{Cu}(\mathrm{II})$. The polyamide as well as Schiff base complexes were characterized by elemental analysis, IR spectroscopy, and magnetic susceptibility measurements. Thermal stabilities of ligand and its various complexes were compared by thermogravimetric analysis.
\end{abstract}

\section{Introduction}

Polyamides, the condensation polymers of dicarboxylic acids and diamines, are frequently referred to as nylons, an important group of polymers which can be synthesized using phosphorylation technique [1]. Oligomers based on these polyamides having reactive terminals are of great importance in order to yield materials with novel properties. Most of the polyamides do not withstand elevated temperature for a longer time. We have tried to get thermally resistant polyamide-metal complexes by inserting metal ions in ligands through the formation of Schiff's base complexes. We have reported Schiff base complexes with oligomeric poly(methylene diphenylene terephthalamide) [2]. In the present communication we are reporting Schiff base complexes of amino-terminated low molecular weight poly(tetramethylene terephthalamide), PTTA, with salicylaldehyde and 2-hydroxy-1-naphthaldehyde complexes of $\mathrm{Co}(\mathrm{II}), \mathrm{Ni}(\mathrm{II})$, and $\mathrm{Cu}(\mathrm{II})$.

Various Schiff bases involving aldehydes with aromatic diamines are extensively studied by numerous workers and are critically reviewed and reported for their biological properties, such as antibacterial and antifungal activities [3-5]. Few polymeric complexes with Schiff's base structures have been synthesized from aromatic amines and phenolic aldehydes. These metal complexes have been widely studied because they have anticancer and herbicidal applications [6-8].

\section{Materials and Methods}

2.1. Materials. The materials of analytical quality or equivalent grades were used. Salicylaldehyde was from S. Merck., India, and 2-hydroxy-1-naphthaldehyde (AG) was from Fluka. 1,4-diaminobutane of AR grade was obtained from E. Merck, India, and was used without further purification. Dimethyl formamide (DMF), dimethyl sulphoxide (DMSO), and pyridine were GR grade products of Qualigens, Glaxo, India, and were distilled before use. Acetates of cobalt(II), nickel(II), and copper(II) (all of AR grade from E. Merck, India) were used as such.

2.2. Synthesis of Amino-Terminated Oligomeric Polyamide (PTTA). The amino terminated oligomeric polyamide ligands were prepared by a known procedure. Terephthalic acid $(0.1 \mathrm{moL})$ was treated with $0.12 \mathrm{moL}$ of 1,4 -diaminobutane, which was finely powdered in the presence of triphenyl phosphite. A mixture of DMF $(150 \mathrm{~mL})$ and pyridine $(30 \mathrm{~mL})$ was taken as solvent. The resulting mixture was maintained 
at $80^{\circ} \mathrm{C}$ for $1.5 \mathrm{hrs}$ under nitrogen atmosphere. The amino group terminated oligomer thus formed was separated out by precipitating out from the reaction mixture by addition of excess of distilled methanol. The precipitated products were washed several times with methanol, filtered under suction, and then dried in air oven at $80^{\circ} \mathrm{C}$ for $20 \mathrm{hrs}$ (see Scheme 1).

The PTTA oligomer was characterized by IR spectroscopy and elemental analysis. The mass average molecular mass $\bar{M}_{w}$ was calculated by viscometery using the following equation given by Aoki et al. [9]:

$$
[\eta]=9.9 \times 10^{-4} \bar{M}_{w} 0.8 .
$$

2.3. Synthesis of Metal-Aldehyde Complexes. The metalaldehyde complexes were prepared by mixing aqueous solutions of acetates of cobalt(II), nickel(II), and copper(II) with ethanolic solutions of salicyladehyde or 2-hydroxy-1naphthaldehyde in respective preparations (see Schemes 2 and 3). The reaction mixture was refluxed with continuous stirring for 2-3 hrs. The coloured solid complexes were filtered, washed with ethanol followed by ether, and then dried in an oven at $60^{\circ} \mathrm{C}$. The complexes were characterized by elemental analysis and IR spectroscopy (see Tables 1 and 2).

2.4. Synthesis of Polyamide-Metal Complexes. Equimolar solutions of metal aldehyde and the amino-terminated oligomer PTTA in $50 \mathrm{~mL}$ hot DMF were mixed in respective preparations and refluxed on a heating mantle for 6$8 \mathrm{hrs}$ to obtain solid coordination polymers. The coloured precipitates were filtered, washed repeatedly with hot DMF and alcohol, and then dried. The polyamide PTTA-metal complexes were coloured, powdery solids insoluble in water and common organic solvent. The reaction scheme for the synthesis of amide-metal complexes is given in Figure 1.

2.5. Characterization. Elemental analysis of polyamide ligand (PTTA) and its coordination polymers was carried out on Perkin Elmer Elemental Analyzer model 2400. The metal contents were determined by complexometric titration with EDTA after decomposing the coordination polymers with fuming nitric acid. Molecular weights of the coordination polymers could not be determined due to their insoluble nature. IR spectra of the ligand and the coordination polymers were recorded on a Perkin Elmer Infrared Spectrophotometer Model 1430 using CsI pellets in 4000$200 \mathrm{~cm}^{-1}$ range. The magnetic susceptibility measurements were done at room temperature with a guoy's balance using $\mathrm{HgCo}(\mathrm{NCS})_{4}$ as a calibrant. Thermogravimetric analysis was performed on a Perkin-Elmer Thermal Analyzer TGA 7 with professional computer 7700 in an inert atmosphere at a heating rate of $20^{\circ} \mathrm{C} / \mathrm{min}$.

\section{Results and Discussion}

The analytical data for the ligand and their metal complexes are presented in Table 1. The compositions and theoretical analytical results are based on assumed structures. In case of PTTA the number of repeated units $(n)$ is taken as 8 to 9 for the theoretical calculations. The average of molecular masses

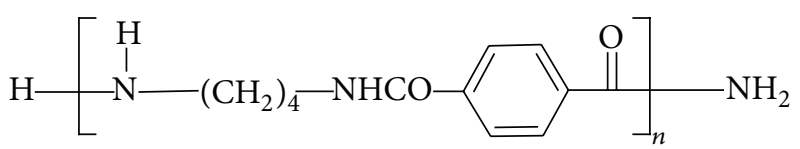

PTTA

SCHEME 1

TABLE 1: Analytical data of PTTA and its metal complexes.

\begin{tabular}{|c|c|c|c|c|c|}
\hline \multirow{2}{*}{ No. } & \multirow{2}{*}{ Compound } & \multicolumn{4}{|c|}{ Elemental analysis* } \\
\hline & & $\% \mathrm{C}$ & $\% \mathrm{H}$ & $\% \mathrm{~N}$ & $\% \mathrm{M}$ \\
\hline \multirow{2}{*}{1} & \multirow{2}{*}{ PTTA } & 63.40 & 5.96 & 13.20 & - \\
\hline & & 64.70 & 6.50 & 14.10 & - \\
\hline \multirow{2}{*}{2} & \multirow{2}{*}{ PTTA-Co(II)-Sal' } & 60.36 & 6.19 & 9.69 & 3.00 \\
\hline & & 62.40 & 5.90 & 11.80 & 2.80 \\
\hline \multirow{2}{*}{3} & \multirow{2}{*}{ PTTA-Ni(II)-Sal' } & 59.86 & 4.97 & 9.22 & 3.22 \\
\hline & & 62.40 & 5.9 & 11.8 & 2.79 \\
\hline \multirow{2}{*}{4} & \multirow{2}{*}{ PTTA-Cu(II)-Sal' } & 60.14 & 5.02 & 10.04 & 3.78 \\
\hline & & 62.20 & 5.88 & 11.79 & 3.00 \\
\hline \multirow{2}{*}{5} & \multirow{2}{*}{ PTTA-Co(II)-Naphthal' } & 62.25 & 4.97 & 9.98 & 3.28 \\
\hline & & 64.60 & 5.77 & 11.03 & 2.68 \\
\hline \multirow{2}{*}{6} & \multirow{2}{*}{ PTTA-Ni(II)-Naphthal' } & 63.98 & 4.88 & 10.12 & 4.00 \\
\hline & & 64.62 & 5.77 & 11.03 & 2.66 \\
\hline \multirow{2}{*}{7} & \multirow{2}{*}{ PTTA-Cu(II)-Naphthal' } & 62.46 & 5.16 & 10.06 & 4.58 \\
\hline & & 64.41 & 5.76 & 10.99 & 2.88 \\
\hline
\end{tabular}

${ }^{*}$ In every case the upper value represents the observed results, and the lower value represents the theoretically calculated values.

calculated on the basis of $n=8$ is $\bar{M}_{w}=1750$ which is close to the experimentally found value that is 1758 .

It was observed that the deviations of the experimental values of elemental analysis from the theoretical ones were beyond experimental error. This discrepancy between the calculated and observed results may be due to the strong moisture pickup tendency of polyamide [10].

Puffer and Sebenda [11] have described sorption and desorption isotherms of water for several crystalline as well as amorphous polyamides and the model amides. They have shown that, in nylon 6, three molecules of water are sorbed on two neighbouring amide groups. The first molecule is firmly bound between the CO groups, and the other two molecules form $\mathrm{H}$-bonds between the $\mathrm{CO}$ and the $\mathrm{NH}$ groups (loosely bound water). In nylons, two to six water molecules are sorbed on two amide groups, probably as firmly bound water. Nylon 7 stands somewhere between the two groups.

In our report on poly(methylene diphenylene terphthalamide) and its coordination polymers, we have also observed the moisture pickup by the polyamide as well as its coordination polymers. The number of water molecules sorbed to the amide groups has been considered as 1.5 per $\mathrm{CONH}$ group excluding the capillary condensation. Because of the polymeric nature, the possibility of structural defects is always there. Further, the exact amount of adsorbed and capillary condensation water cannot be calculated. 


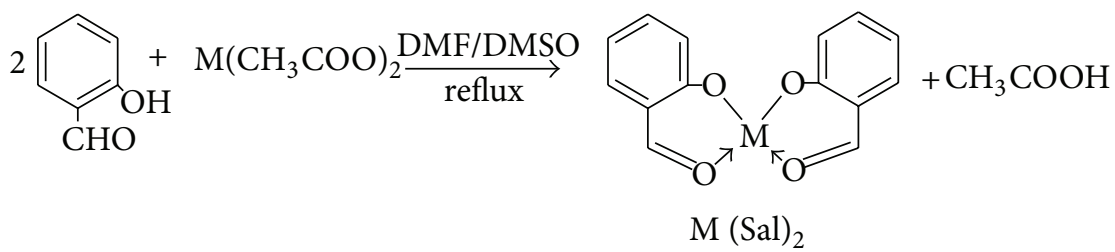

where $\mathrm{M}=\mathrm{Co}(\mathrm{II}), \mathrm{Cu}(\mathrm{II})$, or $\mathrm{Ni}(\mathrm{II})$ and

Sal $=$ salicylaldehyde

SCHeme 2

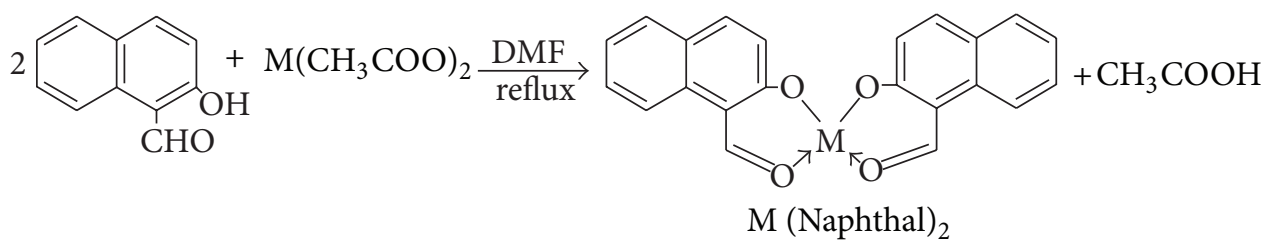

where $\mathrm{M}=\mathrm{Co}(\mathrm{II}), \mathrm{Cu}(\mathrm{II})$, or $\mathrm{Ni}(\mathrm{II})$ and

Naphthal = 2-hydroxy-1-naphthaldehyde

SCHEME 3

3.1. IR Spectral Studies. The structurally important infrared spectral bands for the amino terminated oligomeric ligands and their metal complexes are depicted in Table 2. The assignments are based on the available literature $[12,13]$. In the spectrum of PTTA ligand, there is a sharp and strong band at $3305 \mathrm{~cm}^{-1}$ attributed to $\mathrm{C}=\mathrm{O}$ of amide group of polyamide chain. The band at $1630 \mathrm{~cm}^{-1}$ is attributed to carbonyl frequency of amide I. The absorption due to $\mathrm{NH}$ deformation appears at $1545 \mathrm{~cm}^{-1}$ or amide II. Another band due to $\mathrm{C}-\mathrm{N}$ appears at $1285 \mathrm{~cm}^{-1}$; p-substituted benzene ring vibration appears at $855 \mathrm{~cm}^{-1}$. A band at $1470 \mathrm{~cm}^{-1}$ is assigned to- $\mathrm{CH}_{2}$ bending mode of polyamide chain of PTTA.

The spectra of all the complexes show a strong and sharp band at $3305 \mathrm{~cm}^{-1}$ due to the $\mathrm{NH}$ of polyamide chain of PTTA. Appearance of bands in the region $1550-1555 \mathrm{~cm}^{-1}$, as a result of splitting of amide or $\mathrm{NH}$ deformation in the spectra of all the complexes, indicates involvement of amino group in the complexation. Therefore, one band is ascribed to $\mathrm{C}=\mathrm{N}-\mathrm{M}$ while the other may be due to amide or $\mathrm{NH}$ deformation of amide group of PTTA ligand.

The appearance of new bands in the lower frequency region due to $\mathrm{M}-\mathrm{O}$ at $560-520 \mathrm{~cm}^{-1}$ and due to $\mathrm{M}-\mathrm{N}$ in the region of $480-420 \mathrm{~cm}^{-1}$ further supports and confirms the involvement of nitrogen and oxygen atoms in complex formation.

The other bands of the ligands appear uninfluenced upon coordination suggesting noninvolvement of amide groups of polyamide in coordination. The characteristic absorptions due to acetate group could not be observed in the IR spectra of metal complexes thereby suggesting absence of acetate ions. This observation supports the suggested formula without acetate ions on the basis of elemental analysis data. The appearance of new bands in the far IR region attributed to
$\mathrm{M}-\mathrm{O}$ and $\mathrm{M}-\mathrm{N}$ further confirms the complexation through nitrogen and oxygen.

3.2. Magnetic Susceptibility Measurements. The diamagnetic behaviour of $\mathrm{Ni}$ (II) complexes indicates their square-planar geometry [14]. The square-planar geometry of $\mathrm{Cu}$ (II) complexes is indicated by the magnetic moment values in the range of 1.69-1.85 BM $[15,16]$.

Tetrahedral geometry of $\mathrm{Co}(\mathrm{II})$ complexes is inferred from the magnetic moment values of 4.59 and 4.44 for PTTA-Co(II)-Sal and PTTA-Co(II)-Naphthal, respectively. Diamagnetic behaviour of $\mathrm{Ni}$ (II) complexes indicates their square-planar behaviour. PTTA-Cu(II)-Sal and PTTA$\mathrm{Cu}$ (II)-Naphthal complexes have magnetic moment values of 1.72 and 1.78 , respectively, which were in the range predicted for square-planar complexes of $\mathrm{Cu}(\mathrm{II})$.

On the basis of all the aforementioned studies, a tentative structure as shown in Figure 1 can be proposed for the complexes. Magnetic moment values of amide-metal complexes are summarized in Table 3.

3.3. Thermogravimetric Analysis. Thermogravimetric data of polyamide ligand PTTA and its metal complexes are presented in Table 4. The TG curves of ligand PTTA and its complexes were compared in order to determine the influence of metal ion on the thermal stability. Thermal stabilities have been compared on the basis of initial decomposition temperature, residual percentage by weight at a particular temperature, and energy of activation (E) calculated by Fuoss method [17]. PTTA and its complexes with Ni(II)-Sal and $\mathrm{Cu}$ (II)-Sal show stepwise degradation whereas PTTA-Co(II)Sal complex exhibits continuous degradation above $250^{\circ} \mathrm{C}$, and the weight becomes constant above $550^{\circ} \mathrm{C}$ in all the cases. Residual percentage weights at $600^{\circ} \mathrm{C}$ for the complexes are 
TABLE 2: IR spectral assignments of amides and their metal complexes.

\begin{tabular}{|c|c|c|c|c|c|c|}
\hline \multirow{2}{*}{ Compounds } & \multicolumn{6}{|c|}{ Assignments $\mathrm{cm}^{-1}$} \\
\hline & ${ }^{v} \mathrm{NH}$ & Amide (I) & Amide (II) & ${ }^{v} \mathrm{C}=\mathrm{N}$ & ${ }^{v} \mathrm{M}-\mathrm{O}$ & ${ }^{v} \mathrm{M}-\mathrm{N}$ \\
\hline PTTA & $3305 s$ & $1630 \mathrm{~s}$ & $1545 \mathrm{~s}$ & - & - & - \\
\hline PTTA-Co(II)-Sal' & $3305 s$ & $1630 s$ & $1545 s$ & $1550 \mathrm{~s}$ & $560 \mathrm{~s}$ & $480 \mathrm{~m}$ \\
\hline PTTA-Co(II)-Naphthal' $^{\prime}$ & $3305 s$ & $1630 s$ & $1540 \mathrm{~s}$ & $1550 \mathrm{~s}$ & $560 \mathrm{~s}$ & $485 \mathrm{w}$ \\
\hline PTTA-Ni(II)-Sal' & $3305 s$ & $1630 \mathrm{~s}$ & $1540 \mathrm{~s}$ & $1550 \mathrm{sh}$ & $540 \mathrm{~s}$ & $485 \mathrm{~s}$ \\
\hline PTTA-Ni(II)-Naphthal' $^{\prime}$ & $3305 s$ & $1630 s$ & $1535 s$ & $1550 \mathrm{~s}$ & $570 \mathrm{~s}$ & $470 \mathrm{~m}$ \\
\hline PTTA-Cu(II)-Sal ${ }^{\prime}$ & $3305 s$ & $1630 s$ & $1530 \mathrm{~s}$ & $1555 \mathrm{~s}$ & $520 \mathrm{~s}$ & $490 \mathrm{~s}$ \\
\hline PTTA-Cu(II)-Naphthal' $^{\prime}$ & $3305 s$ & $1630 \mathrm{~s}$ & $1535 s$ & $1560 \mathrm{~s}$ & $560 \mathrm{~m}$ & $480 \mathrm{~s}$ \\
\hline
\end{tabular}

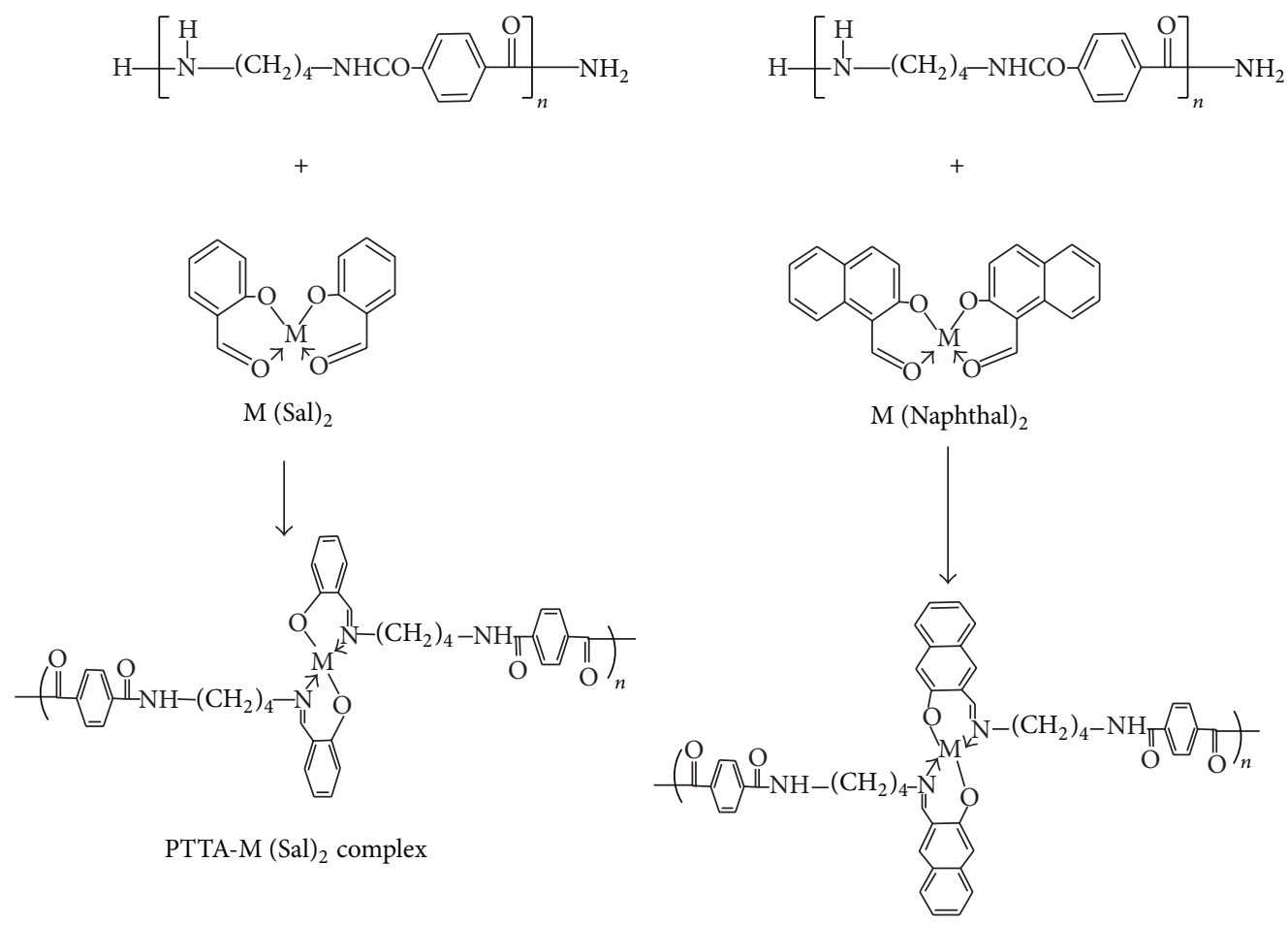

PTTA-M (Naphthal) $)_{2}$ complex

where $\mathrm{M}=\mathrm{Co}(\mathrm{II}), \mathrm{Cu}(\mathrm{II})$, or $\mathrm{Ni}(\mathrm{II})$,

$\mathrm{Sal}=$ salicyladehyde,

and Naphthal = 2-hydroxy-1-naphthaldehyde

FIGURE 1

TABLE 3: Magnetic moment values for polyamide-metal complexes.

\begin{tabular}{lcc}
\hline Compounds & $\begin{array}{c}\text { Magnetic moment } \\
\mu \text { B.M. }\end{array}$ & $\begin{array}{c}\text { Geometry } \\
\text { proposed }\end{array}$ \\
\hline PTTA-Co(II)-Sal $^{\prime}$ & 4.59 & Tetrahedral \\
PTTA-Co(II)-Naphthal $^{\prime}$ & 4.44 & Tetrahedral \\
PTTA-Ni(II)-Sal' $^{\prime}$ & Diamagnetic & Square planar \\
PTTA-Ni(II)-Naphthal $^{\prime}$ & Diamagnetic & Square planar \\
PTTA-Cu(II)-Sal $^{\prime}$ & 1.72 & Square planar \\
PTTA-Cu(II)-Naphthal $^{\prime}$ & 1.78 & Square planar \\
\hline
\end{tabular}

slightly greater than the residual weight for the ligand. The order of stability on the basis of residual weights can be given as

$$
\begin{aligned}
{\text { PTTA-Ni (II) }- \text { Sal }^{\prime}} & >\text { PTTA-Co (II) }- \text { Sal }^{\prime} \\
& >\text { PTTA-Cu (II) }- \text { Sal }^{\prime}>\text { PTTA. }
\end{aligned}
$$

On the basis of energy of activation calculated by Fuoss method, the following order of thermal stability was observed:

$$
\begin{aligned}
{\text { PTTA-Cu (II) }- \text { Sal }^{\prime}}>\text { PTTA-Ni (II) }- \text { Sal }^{\prime} & \\
& >\text { PTTA-Co (II) }- \text { Sal }^{\prime}>\text { PTTA. }
\end{aligned}
$$


TABLE 4: TGA data of PTTA and its metal complexes.

\begin{tabular}{lcccccccc}
\hline \multirow{2}{*}{ Compounds } & \multicolumn{8}{c}{ Temperature in ${ }^{\circ} \mathrm{C}$} \\
& 100 & 200 & 300 & 400 & 500 & 600 & 650 & 700 \\
\hline PTTA & 1.0 & 5.0 & 10.0 & 50.0 & 80.0 & 98.0 & - & - \\
PTTA-Co(II)-Sal' & 0.0 & 0.0 & 0.0 & 19.0 & 72.0 & 92.0 & 93.0 & 94.0 \\
$\begin{array}{l}\text { PTTA-Co(II)- } \\
\text { Naphthal' }\end{array}$ & 2.0 & 3.0 & 7.0 & 25.0 & 91.0 & 92.0 & 92.0 & 92.0 \\
$\begin{array}{l}\text { PTTA-Ni(II)-Sal' } \\
\text { PTTA-Ni(II)- }\end{array}$ & 0.0 & 2.0 & 8.0 & 42.0 & 85.0 & 90.0 & 91.0 & 92.0 \\
$\begin{array}{l}\text { Naphthal' } \\
\text { PTTA-Cu(II)-Sal }\end{array}$ & 4.0 & 7.0 & 10.0 & 22.0 & 69.0 & 76.5 & 78.0 & 79.0 \\
$\begin{array}{l}\text { PTTA-Cu(II)- } \\
\text { Naphthal' }\end{array}$ & 0.0 & 7.0 & 9.0 & 39.0 & 77.0 & 94.0 & 94.0 & 94.5 \\
\hline
\end{tabular}

The expected enhancement in the stabilities could not be obtained in the present case contrary to our previous observation $[18,19]$. It may be due to the fact that sometimes the metal ion catalyses the degradation of the polymeric ligand. It is also possible that slight changes in polymeric environment influence the thermal degradation phenomenon to a large extent. The ligands which show two-step decomposition indicate the formation of intermediate products during thermal degradation process due to the rupture of polyamide chain. The degradation of amide takes place through the random scission of polymer chain into low molecular weight organic compounds and various volatile gaseous products like $\mathrm{CO}_{2}$, $\mathrm{NH}_{3}$, and water are evolved. Therefore it is expected that the different chain environments may be responsible for the different degradation mechanisms. Sazanov et al. [20] in their comparative thermal analysis (CTA) studies with thermally stable polyimides and model compounds have shown that the process of thermooxidation is of successive character; that is, the defective fragments are "burned out" before the main structure is oxidized.

\section{Conclusion}

Introduction of metal ion in polyamide chain through terminal groups generally results in increase in thermal stability of resulting polymer complexes. Further, this work can be extended to prepare similar kinds of polyamides, and, technique may also be used for increasing the thermal stability of fibres, films, sheets this and blocks of amino group containing polyamides.

Slight lowering in the initial decomposition of few complexes than the ligand PTTA may be due to the catalytic effect of metal ion on thermal degradation process.

Another application of these polyamide complexes may be as antimicrobial agents because of Schiff base moiety.

\section{References}

[1] U. D. N. Bajpai, S. Rai, and A. Bajpai, "Synthesis and characterization of metal-containing coordination polymers of poly (methylene diphenylene terephthalamide)," Journal of Applied Polymer Science, vol. 48, no. 7, pp. 1241-1248, 1993.
[2] U. D. N. Bajpai, A. Bajpai, and S. Rai, "Heat resistant coordination polymers based on amino group terminated oligomer of hexamethyleneadipamide," Synthesis and Reactivity in Inorganic and Metal Organic Compounds, vol. 24, no. 10, pp. 1719-1732, 1994.

[3] C. Selvi and D. Nartop, "Novel polymer anchored Cr(III) Schiff base complexes: synthesis, characterization and antimicrobial properties," Spectrochimica Acta A, vol. 95, pp. 165-171, 2012.

[4] N. Nishat, R. Rasool, S. Parveen, and S. A. Khan, "New antimicrobial agents: the synthesis of Schiff base polymers containing transition metals and their characterization and applications," Journal of Applied Polymer Science, vol. 122, no. 4, pp. 27562764, 2011.

[5] A. K. Kaura and M. Kaura, "Synthesis, spectral and comparative antimicrobial study of schiff bases," International Journal of Chemical and Pharmaceutical Sciences, vol. 3, no. 4, pp. 24-29, 2012.

[6] P. L. Maurya, B. V. Agarwala, and A. K. Dey, "Condensation polymerization of bis-(salicylaldehydo) metal(II) with diamines," Polymer Bulletin, vol. 3, no. 5, pp. 253-259, 1980.

[7] A. Prakash and D. Adhikari, "Application of Schiff bases and their metal complexes-a review," International Journal of ChemTech Research, vol. 3, no. 4, pp. 1891-1896, 2011.

[8] S. Kumar, D. N. Dhar, and P. N. Saxena, "Applications of metal complexes of Schiff bases-a review," Journal of Scientific and Industrial Research, vol. 68, no. 3, pp. 181-187, 2009.

[9] H. Aoki, D. R. Coffin, T. A. Hancock et al., "Synthesis, characterization, rheological, and fiber formation studies of p-linked aromatic polyamides," Journal of Polymer Science Polymer Symposium, no. 65, pp. 29-40, 1978.

[10] Y. P. Khanna, E. M. Pearce, B. D. Forman, and D. A. Bini, "Aromatic polyamides-1. Synthesis and characterization of some aromatic polyamides and their model diamides," Journal of Polymer Science A, vol. 19, no. 11, pp. 2799-2816, 1981.

[11] R. Puffer and J. Sebenda, "On the structure and properties of polyamides. XXVII. The mechanism of water sorption in polyamides," Journal of Polymer Science C, vol. 16, p. 79, 1977.

[12] J. Bellamy, The Infrared Spectra of Complex Molecules, John Wiley \& Sons, New York, NY, USA, 1964.

[13] N. B. Colthup, L. H. Daly, and S. E. Wiberly, Introduction To Infrared and Raman Spectroscopy, Academic Press, New York, NY, USA, 1969.

[14] E. K. Barefield, D. H. Busch, and S. M. Nelson, "Cobalt and nickel complexes having anomalous magnetic moments," Quarterly Reviews, vol. 22, p. 457, 1968.

[15] H. B. Gray and C. J. Balhaueen, "A molecular orbital theory for square planar metal complexes," Journal American Chemical Society, vol. 85, p. 260, 1963.

[16] W. E. Hatfield, Y. Muto, H. B. Jonassen, and J. S. Paschal, "Substituted pyridine N-oxide complexes of copper(II) halides," Inorganic Chemistry, vol. 4, no. 1, pp. 97-99, 1965.

[17] R. M. Fuoss, I. O. Salyer, and H. S. Wilson, "Evaluation of rate constants from thermogravimetric data," Journal of Polymer Science, vol. A2, p. 3147, 1962.

[18] U. D. N. Bajpai, A. Bajpai, and M. Khandwe, "Synthesis and characterization of poly(thiooxamide) metal complexes," in Metal Containing Polymeric Materials, C. U. Pittman, Ed., Plenum Publishing Corporation, New York, NY, USA, 1996. 
[19] J. H. Hodgkin and H. Heller, "Synthesis of conjugated Schiffbase polymers," Journal of Polymer Science C, vol. 29, pp. 37-46, 1970.

[20] Y. N. Sazanov, L. A. Shibaev, and T. A. Antonova, "Comparative thermal analysis (CTA) of thermally-stable polymers and model compounds. Polyimides and model compounds," Journal of Thermal Analysis, vol. 18, no. 1, pp. 65-75, 1980. 

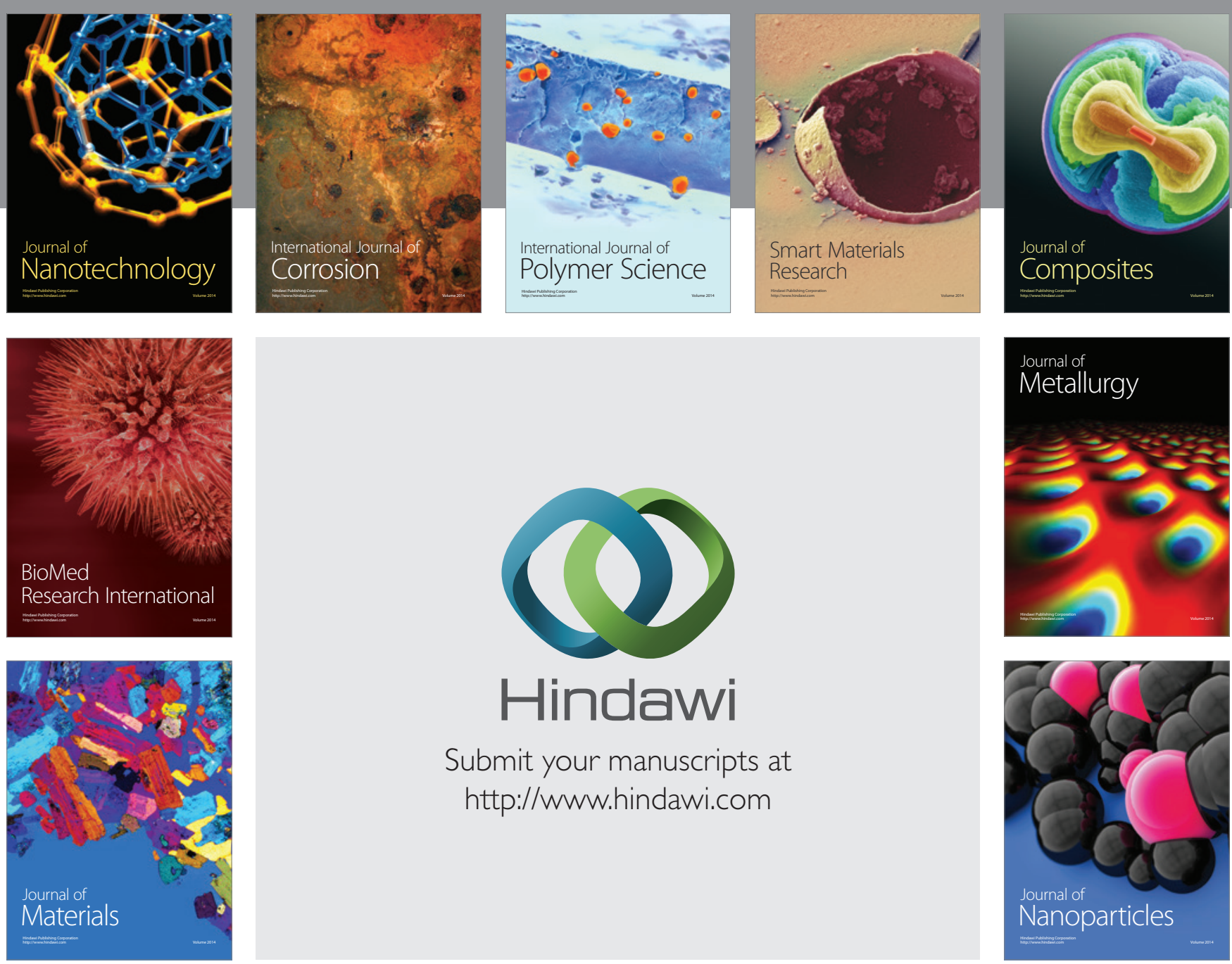

Submit your manuscripts at http://www.hindawi.com
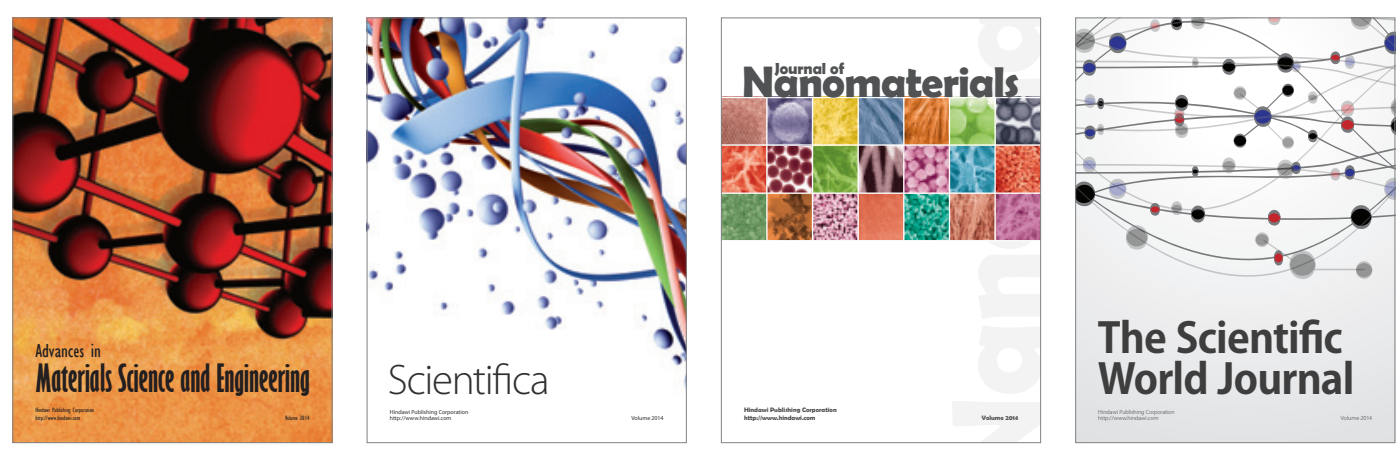

\section{The Scientific World Journal}
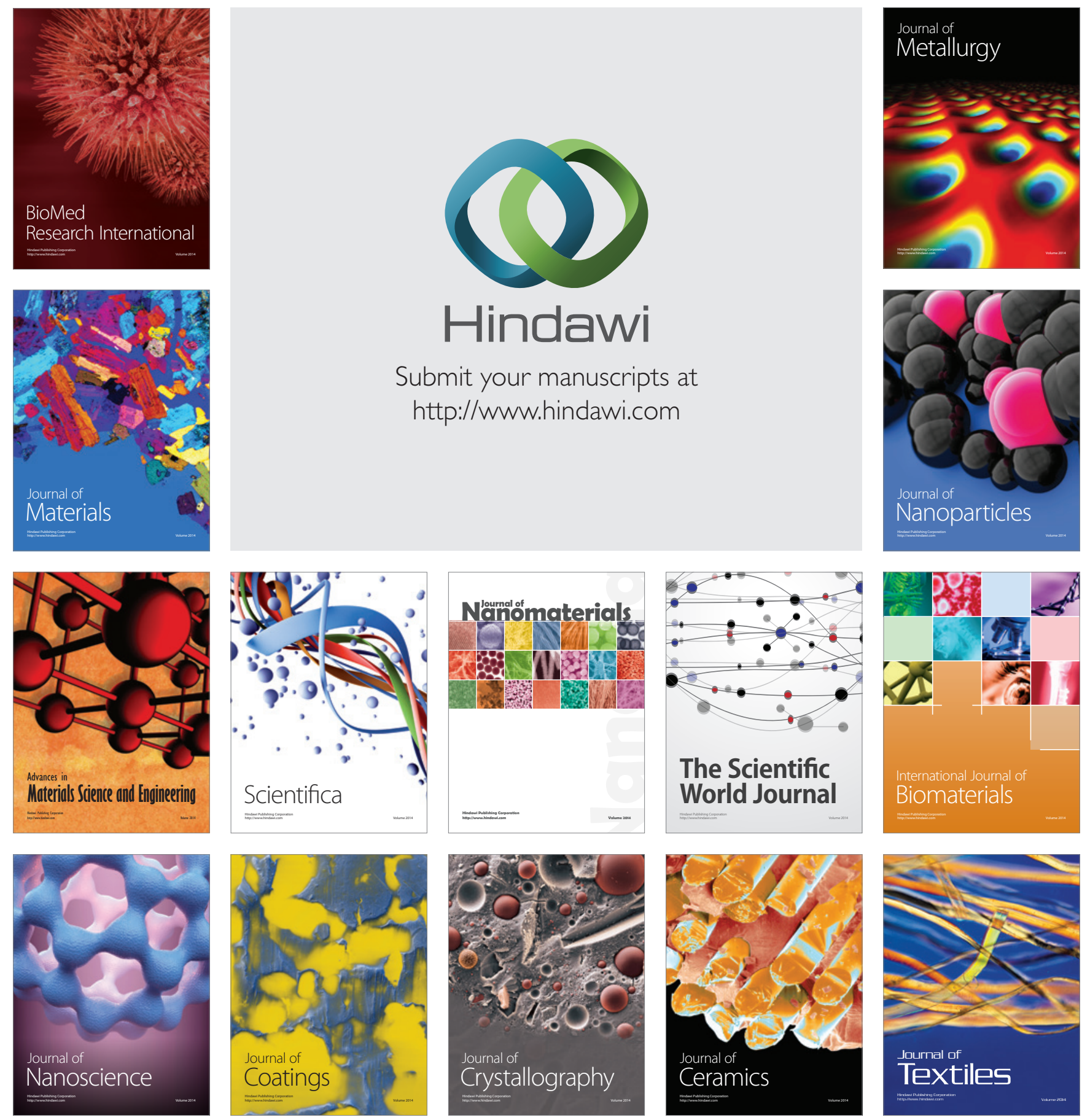